\title{
Fractal Dimension Analysis of Pipelines Thermograms for Hidden Defects
}

\section{Detection}

\author{
B.B. Banduryan, V.F. Klepikov, V.V. Lytvynenko and O.A. Startsev \\ Institute of Electrophysics and Radiation Technologies, National Academy of Sciences of \\ Ukraine, Chernyshevsky St. 28, PO Box 8812, 61002 Kharkiv, Ukraine \\ startsev-alex@ukr.net
}

Experimental and numerical studies aiming to enhance the defects detection in industrial pipelines were conducted. The stainless steel pipeline sample with a thickness of $4 \mathrm{~mm}$ and a 219-mm-diameter was used. Its external surface was smooth and cleared form paint. A few hidden defects were made on its internal surface to simulate electrochemical corrosion and fluid flow. We made a shallow longitudinal groove with pits of different penetration depth in the pipe wall. Typical alternating current $(220$ Volts, $5 \mathrm{~A}, 50 \mathrm{~Hz})$ was applied to heat the prepared sample during $38 \mathrm{~min}$. Infrared monitoring of steady heating and cooling processes was carried out using the thermographic imager Fluke Ti32. The detected peak temperature was around $323 \mathrm{~K}$, compared to the initial temperature of $287 \mathrm{~K}$. Thermograms were taken every minute.

To reveal the low-resolution changes in heat propagation, which accompanied the defects, we examined the fractal dimension distributions of the obtained thermographic data sets. The fractal dimension values were statistically calculated using the classic Clarke's triangular prism surface area method, the modified Clarke's method proposed by Qiu, eight-pixel, maxdifference and mean-difference methods introduced by Sun. We used both the step size squared as well as the step size in the log-log regression line. The step size increment was variable: arithmetic, geometric or divisor-step. The geometric-square frames with variable side length were employed over all data sets with the unary moving step for calculation the local fractal dimension.

It was found, the hidden defects can be localized using the fractal post-processing of thermographic data sets. Filtering of the fractal dimension maps and fractal gradients of thermograms provides more accurate segmentation of the thermal inhomogeneities, especially caused by the artificial defects, than direct filtering of thermograms by temperature values. Unusually, it helps to separate the corrosion-like hidden defects on the internal surface from the as-fabricated imperfections (rollings, etc). It was proven, the fractal mapping significantly decrease blurring. The main useful information lies in the long tail of the lognormal-like fractal dimensions distribution, and using its peaks we can distinguish some thermal irregularity. However, the fractal dimensions spectra are quite sensitive to the chosen calculation procedure and the imager settings. Square frame shape adds some distortions in the fractal dimensions maps as well, so these aspects need to be clarified in further studies.

Keywords: pipeline, hidden defect, fractal mapping. 